\title{
Comportamento reológico e atividade antimicrobiana de uma formulação tópica contendo extrato de Heliotropium indicum $\mathbf{L}$.
}

\author{
Rheological behavior and antimicrobial activity of a topical formulation containing extract of \\ Heliotropium indicum $\mathrm{L}$. \\ Comportamiento reológico y actividad antimicrobiana de una formulación tópica que contiene \\ extracto de Heliotropium indicum $\mathbf{L}$.
}

Recebido: 14/04/2021 | Revisado: 21/04/2021 | Aceito: 23/04/2021 | Publicado: 08/05/2021

\author{
Russany Silva da Costa \\ ORCID: https://orcid.org/0000-0002-2705-1591 \\ Universidade Federal do Pará, Brasil \\ E-mail: russany@ufpa.br \\ Roseane Maria Ribeiro Costa \\ ORCID: https://orcid.org/0000-0001-5470-0617 \\ Universidade Federal do Pará, Brasil \\ E-mail: roseribeiro01@yahoo.com.br \\ José Otávio Carréra Silva Júnior \\ ORCID: https://orcid.org/0000-0003-1691- 1039 \\ Universidade Federal do Pará, Brasil \\ E-mail: carrera@ufpa.br
}

\begin{abstract}
Resumo
A espécie vegetal Heliotropium indicum L. (Boraginaceae) é utilizada na forma de cataplasma para tratar regiões inflamadas no corpo, devido suas propriedades anti-inflamatória e cicatrizante. Formulações tópicas apresentam inúmeras vantagens para o tratamento de lesões cutâneas, entre elas destaca-se a promoção do efeito local do fármaco quando sua distribuição sistêmica não é desejada. Objetivo: Avaliar o comportamento reológico e a atividade antimicrobiana de uma formulação semissólida para uso tópico contendo o extrato de $H$. indicum. Metodologia: Para obtenção do gel, foi desenvolvido uma formulação contendo hidroxietilcelulose $(1,5 \%)$ e o extrato. A formulação foi submetida ao estudo da estabilidade preliminar, de acordo com o guia de estabilidade de produtos cosméticos (ANVISA), comportamento reológico e atividade antimicrobiana através do método de disco-difusão em meio sólido. A formulação foi submetida em estufa $\left(45 \pm 2^{\circ} \mathrm{C}\right)$ e a temperatura ambiente $\left(25 \pm 2^{\circ} \mathrm{C}\right)$, para avaliação do estresse térmico. As leituras do comportamento reológico do gel foram mensuradas em velocidade crescente e decrescente pela velocidade versus tensão de cisalhamento e pela viscosidade aparente versus velocidade de cisalhamento. Resultados: Não houve desestruturação da cadeia polimérica do gel e a formulação apresentou o comportamento de material pseudoplástico e tixotrópico, com atividade antimicrobiana frente cepas de Staphylococcus aureus (ATCC 29913), nas concentrações de 500 e $250 \mathrm{mg} / \mathrm{mL}$. Conclusão: A formulação manteve-se estável, apresentando um decréscimo na viscosidade aparente com aumento da taxa de cisalhamento, comum de sistemas contendo polímeros em dispersão, e uma promissora aplicação para tratamento de infecções cutâneas.
\end{abstract}

Palavras-chave: Heliotropium indicum L.; Gel; Estabilidade; Viscosidade; Antimicrobianos.

\begin{abstract}
The vegetable species Heliotropium indicum L. (Boraginaceae) is used as poultice to treat inflamed regions of the body, due to its anti-inflammatory and healing properties. Topical formulations have numerous advantages for the treatment of skin lesions, among them stands out the promotion of the local effect of the drug, when its systemic distribution is not desired. Aim: This study aimed to evaluate the rheological behavior and antimicrobial activity of a semi-solid formulation for topical use containing the extract of $\mathrm{H}$. indicum. Metodology: To developed the gel, the formulation was contained by hydroxyethylcellulose $(1,5 \%)$ and the extract. The formulation was submitted to the preliminary stability study, according to the cosmetic product stability guide (ANVISA), rheological behavior and antimicrobial activity through the disc-diffusion method in solid medium. The formulation was submitted to an oven $\left(45 \pm 2{ }^{\circ} \mathrm{C}\right)$ and at room temperature $\left(25 \pm 2{ }^{\circ} \mathrm{C}\right)$ to evaluate thermal stress. The readings of the rheological behavior of the gel were measured in increasing and decreasing speed versus shear stress by the apparent viscosity versus shear speed. Results: There was no breakdown of the polymeric gel chain and the formulation showed the behavior of pseudoplastic and thixotropic material, with an antimicrobial activity against strains of Staphylococcus aureus (ATCC 29913), at concentrations of 500 and $250 \mathrm{mg} / \mathrm{mL}$. Conclusion: This formulation remained stable, showing a decrease in apparent
\end{abstract}


viscosity with an increase in shear rate, common in systems containing dispersed polymers and a promising application for the treatment of skin infections.

Keywords: Heliotropium indicum L.; Gel; Stability; Viscosity; Antimicrobials.

\section{Resumen}

La especie vegetal Heliotropium indicum L. (Boraginaceae) se utiliza en la forma de cataplasma para tratar regiones inflamadas del cuerpo, debido a sus propiedades antiinflamatorias y cicatrizantes. Las formulaciones tópicas presentan numerosas ventajas para el tratamiento de lesiones cutáneas, como la promoción del efecto local del fármaco cuando no se desea su distribución sistémica. Objetivo: Evaluar el comportamiento reológico y la actividad antimicrobiana de una formulación semisólida para uso tópico que contiene el extracto de $H$. indicum. Metodología: Para la obtención del gel se desarrolló una formulación que contenía hidroxietilcelulosa $(1,5 \%)$ y el extracto. La formulación fue sometida a un estudio preliminar de estabilidad, de acuerdo con la guía de estabilidad del producto cosmético (ANVISA), comportamiento reológico y actividad antimicrobiana mediante el método de difusión por disco en medio sólido. La formulacion se sometieron a estufa $\left(45 \pm 2{ }^{\circ} \mathrm{C}\right)$ y a temperatura ambiente $\left(25 \pm 2{ }^{\circ} \mathrm{C}\right)$, para evaluar el estrés térmico. Las lecturas del comportamiento reológico del gel se midieron a una velocidad creciente y decreciente por la velocidad frente al esfuerzo cortante y por la viscosidad aparente frente la velocidad cortante. Resultados: No hubo ruptura de la cadena de gel polimérico y la formulación mostró el comportamiento de material pseudoplástico y tixotrópico, con la actividad antimicrobiana frente la cepas de Staphylococcus aureus (ATCC 29913), concentraciones de 500 y $250 \mathrm{mg} /$ mL. Conclusión: La formulación se mantuvo estable, mostrando una disminución de la viscosidad aparente con un aumento de la velocidad de cizallamiento, común en sistemas que contienen polímeros dispersos, y una aplicación prometedora para el tratamiento de infecciones cutáneas.

Palabras clave: Heliotropium indicum L.; Gel; Estabilidad; Viscosidad; Antimicrobianos.

\section{Introdução}

Formulações farmacêuticas para aplicação tópica no tratamento de lesões cutâneas apresentam inúmeras vantagens, como: promoção de efeito local quando a distribuição sistêmica não é desejada ou quando promovem efeitos adversos (Corrêa et al., 2005; Dubey et al., 2007), além da preservação da ação do fármaco. Assim, as formulações semissólidas para uso tópico são aquelas que se destinam a aplicação sobre a pele ou mucosa, e do ponto de vista farmacotécnico, as mais utilizadas, são as pomadas, cremes, géis, pastas e emplastros (Ansel et al., 2013).

Nesse contexto, a formulação semissólida (gel) é muito utilizada, pois atua como veículo para medicamentos topicamente administrados através da camada córnea, tecido retal, mucosa nasal, vagina, tecido bucal, membrana uretral e revestimento externo da orelha (Corrêa et al., 2005; Qwist et al., 2019). Devido ao seu comportamento reológico, os géis podem aderir à superfície de aplicação por períodos suficientemente longos até serem removidos, apresentando uma boa adesividade cutânea (Bonfim et al., 2020). Essa propriedade ajuda no prolongamento da liberação do fármaco no local de aplicação, facilitando a aplicação e a capacidade de liberação tópica de uma grande variedade de fármacos (Carvalho et al., 2013; Ferreira et al., 2016). Portanto, as características reológicas são propriedades importantes que devem ser consideradas na fabricação, estocagem e aplicação de produtos para uso tópico. Cada categoria de produto deve apresentar um comportamento reológico adequado à sua aplicação, sendo conveniente conhecer a velocidade de deformação que as formulações estarão sujeitas (Ferreira et al., 2016).

Com esse propósito, os géis hidrossolúveis têm sido muito usados em formulações cosméticas, pois apresentam facilidade de espalhamento, não são gordurosos e podem veicular ingredientes ativos hidrossolúveis. Geralmente, as substâncias formadoras de géis são polímeros, e quando dispersos em meio aquoso assumem a conformação doadora de viscosidade da preparação (Bonfim et al., 2020). Logo, os géis são preparações semissólidas constituídas por dispersões de um sólido (polímero) num líquido formando um excipiente transparente ou translúcido, o qual fornece firmeza a uma solução ou dispersão coloidal (Brasil, 2010).

Ademais, os estudos de estabilidade de produtos cosméticos e farmacêuticos procuram fornecer informações que indiquem o grau de estabilidade relativa de um produto nas condições diversas de exposição a que estão submetidos, até seu prazo de validade. Gerando, portanto, subsídios para a orientação nos estudos de desenvolvimento, como: a escolha dos 
componentes da formulação e do material de acondicionamento adequado; a forma de apresentação; os materiais de acondicionamento e as embalagens alternativas e a confirmação do prazo de validade estimado. Tais estudos consistem na realização de testes na fase inicial do desenvolvimento do produto, empregando-se condições extremas de temperatura (estresse térmico), com o objetivo de acelerar possíveis reações entre seus componentes e o surgimento de sinais de instabilidade que devem ser observados e analisados conforme as características específicas de cada produto (Brasil, 2010; Isaac et al., 2008).

Nesse sentido, os estudos preliminares de estabilidade de uma formulação semissólida ajudam na obtenção de resultados satisfatórios para o desenvolvimento de uma formulação estável, em um curto intervalo de tempo (Lima et al., 2008). Particularmente, nas formulações fitoterápicas do tipo gel, as incompatibilidades podem ocorrer entre o polímero formador do gel e os demais constituintes da formulação, principalmente após a incorporação de extratos vegetais gerando processos de incompatibilidades, como: turbidez, precipitação, cristalização, alteração de cor, alteração de odor e desestruturação da cadeia polimérica (Isaac et al., 2008).

Por isso, o interesse em se estudar a espécie Heliotropium indicum L. (Boraginaceae) deu-se em função aos relatos de que suas folhas são utilizadas, na região amazônica, para tratamento tópico de hemorroidas, afecções cutâneas e em queimaduras. Segundo Guerrero (1994), as folhas de H. indicum L. também são usadas em regiões inflamadas do corpo. Nesse contexto, as propriedades cicatrizante, anti-inflamatória e antimicrobiana podem ser atribuídas a classe de alcaloides pirrolizidínicos e aos taninos, presente em todas as partes do vegetal (Singh et al., 2005).

Embora as indústrias farmacêuticas venham produzindo novos antibióticos e modificando alguns fármacos já existentes, as estatísticas mostram que a resistência a essas moléculas pelos microrganismos está em contínuo crescimento, atingindo níveis perigosamente elevados em todas as partes do mundo (OPAS, 2017). Por isso, novos mecanismos de resistência emergem e se espalham globalmente todos os dias (Marks et al., 2020). Tal fato causa preocupação, pois cresce o número de pacientes em hospitais que apresentam sua imunidade suprimida, concomitantemente ao surgimento de linhagens de bactérias e fungos com novos perfis de resistências aos antimicrobianos disponíveis (Rincón-León \& Navarro-Fuentes, 2016; Bresslau, 2017; Barros et al., 2019; Marks et al., 2020; Stella \& Oliveira, 2020). Ademais, as buscas por novas substâncias com potencial atividade antimicrobiana, provenientes de plantas, veem crescendo consideravelmente nos últimos anos (Barros et al., 2019; Alves et al., 2020). Segundo a OMS, as plantas medicinais são a melhor fonte de obtenção de uma variedade de insumos farmacêuticos ativos e cerca de $80 \%$ da população mundial usa a medicina tradicional buscando o alívio de alguma sintomatologia dolorosa ou desagradável.

Por isso, esse trabalho objetivou avaliar o comportamento reológico, a estabilidade preliminar e a atividade antimicrobiana de um fitocosmético (gel) contendo o extrato de Heliotropium indicum L., visando sua aplicação em tratamentos de lesões cutâneas.

\section{Metodologia}

\subsection{Material}

As folhas da espécie vegetal Heliotropium indicum L. foram coletadas no município de São Miguel do Guamá, à 150 km da capital Belém (PA), com latitude de $01^{\circ} 37^{\prime} 36^{\prime \prime}$ sul e longitude de $47^{\circ} 29^{\prime} 00^{\prime \prime}$ oeste. Sua exsicata foi submetida ao acervo do herbário do Museu Paraense Emílio Goeldi (MG 145574). Para preparação do gel utilizou-se o hidroxietilcelulose (Natrozol® 250 HHRP), como agente doador de viscosidade, propilenoglicol PA-ACS (Química, Erich LDTA), por sua ação umectante e o metilparabeno (Mapric $®)$, conservante.

Os microrganismos testados foram cepas padrão ATCC (American Type Culture Colection) recomendadas para testes de suscetibilidade aos antimicrobianos. Foram utilizadas as cepas de microrganismos: Staphylococcus aureus ATCC 29913; 
Escherichia coli ATCC 25922; Pseudomonas aeruginosa ATCC 25853; Candida albicans ATCC 40175, provenientes do Instituto Oswaldo Cruz - RJ.

\subsection{Métodos}

Esse estudo é de natureza quantitativa, e se caracterizou por um estudo do tipo investigação experimental em laboratório (Estrela, 2018).

\subsubsection{Obtenção do extrato seco por liofilização}

A obtenção do extrato seco da espécie vegetal (H. indicum) foi realizada de acordo com o método preconizado pela Farmacopeia Brasileira (Brasil, 2010), seguindo o processo extrativo geral maceração. O extrato foi obtido a partir de uma solução hidroalcoólica $\left(70^{\circ} \mathrm{GL}\right)$, por 10 dias, com teor de $20 \%$ da droga vegetal (p/v). Para evaporação do solvente orgânico, o extrato foi submetido em rotaevaporador (Fisatrom, modelo 802), sob pressão reduzida e temperatura controlada $\left(40 \pm 2^{\circ} \mathrm{C}\right)$. Posteriormente, o extrato aquoso resultante foi resfriado a $-70^{\circ} \mathrm{C}$ e liofilizado (Liofilizador Thermo Savant, modelo MicroModulyo/115, com bomba a vácuo acoplada LP200-Thermo Savant) e mantido protegido da luz e sob temperatura ambiente $\left(25^{\circ} \mathrm{C}\right)$ até o momento das análises.

\subsubsection{Obtenção da formulação}

Para a preparação da formulação foi empregada uma base galênica com característica hidrofílica utilizando-se o polímero hidroxietilcelulose - HEC (Natrozol® 250 HHRP), para a incorporação do extrato de H. indicum L. (10\%).

A Tabela 1 mostra a composição da formulação contendo o extrato de H. indicum L. No preparo da base gelificante, o conservante (metilparabeno) foi diluído em água destilada e a mistura foi aquecida $\left(55,0 \pm 2,0{ }^{\circ} \mathrm{C}\right)$. Em seguida, foi adicionado o polímero (HEC) umedecido em propilenoglicol. Após a completa dispersão dos componentes da formulação, a mistura foi retirada do aquecimento e deixada em repouso à temperatura ambiente, por 24 horas. Posteriormente, o extrato de $H$. indicum $\mathrm{L}$. foi incorporado à base.

Tabela 1. Componentes da formulação semissólida (gel) contendo extrato de Heliotropium indicum L. em suas respectivas concentrações.

\begin{tabular}{l|c}
\hline Componentes da Formulação & Concentração (\%) \\
\hline Extrato de Heliotropium indicum L. & 10 \\
\hline Hidroxietilcelulose (HEC) & 1,5 \\
\hline Propilenoglicol & 5,0 \\
\hline Metilparabeno & 0,2 \\
\hline Água destilada & 100 \\
\hline
\end{tabular}

q.s.p. = quantidade suficiente para. Fonte: Autores. 


\subsubsection{Avaliação preliminar da estabilidade da formulação}

\subsubsection{Teste de centrifugação}

Foram pesados 5,0 g do gel em tubos de centrífuga (Fanem Ltda-Mod. 206 R, Excelsa BABY II-440 watts). O teste da centrifugação foi realizado em temperatura ambiente $\left(25 \pm 2,0^{\circ} \mathrm{C}\right)$, com velocidade de rotação de $3.000 \mathrm{rpm}$, durante 30 minutos (Brasil, 2004).

\subsubsection{Teste do estresse térmico}

Amostras de gel $(5,0 \mathrm{~g})$ foram separadas em 12 vidros âmbar. Sendo, seis amostras submetidas ao aquecimento (45 \pm $\left.2,0^{\circ} \mathrm{C}\right)$, e avaliadas em 6 ciclos no intervalo de $24 \mathrm{~h}$. Enquanto as demais amostras permaneceram à temperatura ambiente $(25 \pm$ $2,0^{\circ} \mathrm{C}$ ) e foram utilizadas como controle. Após o término de cada ciclo, as amostras foram avaliadas através dos seguintes parâmetros: características organolépticas (enfatizando possíveis alterações na aparência, cor, odor, homogeneidade, brilho, ausência de grumos e precipitados) e determinação do $\mathrm{pH}$. Os resultados obtidos nos tempos $\mathrm{t}_{1}$ a $\mathrm{t}_{6}$ do ciclo da estufa $\left(45^{\circ} \mathrm{C}\right)$ foram comparados aos resultados das amostras controle $\left(25^{\circ} \mathrm{C}\right)(\mathrm{Brasil}, 2004)$.

\subsubsection{Determinação do valor de $\mathrm{pH}$}

$\mathrm{O} \mathrm{pH}$ das amostras do gel contendo o extrato vegetal $\left(\mathrm{t}_{0}\right.$ a $\left.\mathrm{t}_{6}\right)$ foi determinado em dispersões das amostras em água recém destilada na proporção $1: 10(\mathrm{p} / \mathrm{v})$, à $25^{\circ} \mathrm{C}($ Brasil, 2010).

\subsubsection{Avaliação do perfil reológico da formulação}

Após 48h de sua preparação, a formulação foi avaliada quanto ao comportamento reológico em reômetro (Rheotet, modelo 2, tipo cilindro), utilizando dispositivo AIICS. As leituras mensuradas foram realizadas à velocidade crescente $\mathrm{e}$ decrescente para obtenção das curvas ascendentes e descendentes (Silva Júnior \& Pereira, 2009). A partir dos dados experimentais, as curvas de escoamento foram avaliadas graficamente pela velocidade versus tensão de cisalhamento e pela viscosidade aparente versus velocidade de cisalhamento.

\subsubsection{Avaliação da atividade antimicrobiana}

Para a avaliação preliminar da atividade antimicrobiana do gel contendo o extrato de $H$. indicum L., empregou-se o método de disco-difusão em meio sólido (NCCLS, 2003). A placa de petri contendo meio ágar Müeller-Hinton foi inoculada através da técnica da semeadura do swab. Posteriormente procedeu-se a aplicação dos discos contendo o gel nas placas, o qual foi realizado por um conjunto pré-determinado de discos de papel de filtro padronizados e estéreis (Whatman - tipo $\mathrm{n}^{\circ} 3$ ) $\mathrm{com}$ $6,0 \mathrm{~mm}$ de diâmetro. Em cada disco foi impregnado $10 \mu \mathrm{L}$ do gel contendo o extrato de $H$. indicum e dissolvido em DMSO, nas concentrações de 500, 250, 125 e 62,5 mg/mL. As placas, contendo o inócuo semeado, os discos controle (10 $\mu \mathrm{L}$ de DMSO) e a amostra (10 $\mu \mathrm{L}$ do gel contendo o extrato dissolvido em DMSO), foram armazenados em estufa $\left(35,5 \pm 1,0^{\circ} \mathrm{C}\right)$, por 24 horas.

Após a incubação das placas, fez-se a leitura dos resultados referente a formação de halos de inibição, em milímetros, ao redor dos discos contendo a amostra. O resultado final representou a média de duas determinações e foi considerado como suscetível halo igual ou superior a 8,0 mm de diâmetro (NCCLS, 2003; Parekh \& Chanda, 2007).

\subsubsection{Análise Estatística}

Os resultados foram apresentados como média \pm DPR. Para a análise de variância foi considerado um nível de significância de 95\% (Statistica Software ${ }^{\circledR}$ versão 10.0), seguida por múltiplas comparações por Teste de Turkey. 


\section{Resultados e Discussão}

\subsection{Estabilidade preliminar da formulação}

A formulação contendo o extrato de $H$. indicum L. apresentou uma boa consistência gelificante, com coloração verde claro, odor característico do extrato de $H$. indicum e pH variando de 6,6 a 5,1 (Tabela 2). Costa et al. (2011) constaram que houve uma variação do $\mathrm{pH}(7,8 \pm 0,1)$ do extrato de $H$. indicum $\mathrm{L}$. e após sua adição à formulação $(6,3 \pm 0,1)$. Por isso, a escolha do polímero (HEC) ocorreu em função da sua menor sensibilidade a variações de pH do meio. Já o teste de centrifugação mostrou que o gel contendo o extrato vegetal não apresentou sinais de instabilidade, como a desestruturação da cadeia polimérica, assim foi possível prosseguir com as análises posteriores (Tabela 2).

Tabela 2. Características organolépticas do gel contendo extrato de Heliotropium indicum L.

\begin{tabular}{c|c}
\hline Análise & Formulação \\
\hline Aspecto & Gelificado \\
\hline Cor & Levemente verde \\
\hline Odor & Característico \\
\hline $\mathrm{pH}$ & $6,6-5,1$ \\
\hline Centrifugação & Estável \\
\hline
\end{tabular}

Fonte: Autores.

De acordo com o "Guia para Realização de Estudos de Estabilidade de Cosméticos” da Agência Nacional de Vigilância Sanitária (ANVISA) o teste de centrifugação tem caráter eliminatório e foi realizado para avaliação da estabilidade preliminar da formulação (Brasil, 2004). Dessa forma, o gel foi submetido à força centrípeta visando à aceleração de possíveis processos de instabilidade, o que permitiu a continuidade dos teste de estabilidade do gel, uma vez que este parâmetro não assegura sua estabilidade, mas indica que o produto pode ser submetido, sem necessidade de reformulação, ao teste do estresse térmico (Isaac et al., 2008; Lima et al., 2008; Brasil, 2004; Silva \& Torres, 2019).

Ademais, o gel apresentou aparência verde clara (Tabela 2), o que facilita a aceitabilidade pelos pacientes ao tratamento utilizando formulações tópicas, pois pesquisas já demonstraram a preferência dos pacientes em utilizar géis transparentes, uma vez que aqueles com coloração escura causam a impressão de que podem manchar a pele. Com isso, a formulação semissólida permaneceu estável durante o período da análise, pois não foi identificado sinérese, ou seja, a separação espontânea de um sistema coloidal em duas fases: gel e líquido, alteração de cor, odor e da aparência da homogeneidade.

Já na avaliação do pH (Tabela 3), a formulação apresentou uma variação no valor de pH $(6,3 \pm 0,1)$ em comparação ao extrato bruto $(7,8 \pm 0,1)$ (Costa et al., 2011), quando submetido ao estresse térmico. Tal resultado pode ser explicado pela natureza química dos excipientes empregados na formulação. Contudo, a partir do segundo dia de análise $\left(\mathrm{t}_{2}\right)$ o pH da amostra apresentou uma queda significativa, com variação de 5,1 a 5,8 (Tabela 3). Todavia, a diminuição do $\mathrm{pH}$ não interferiu na estabilidade da formulação, uma vez o pH manteve-se aceitável para aplicação tópica $(4,5-5,5)$, corroborando para a redução de irritabilidade da pele, bem como a obtenção de um produto mais estável e seguro para uso (Silva \& Torres, 2019; Silva et al., 2019). Nesse contexto, a ocorrência de variações do $\mathrm{pH}$ cutâneo é dependente de diferentes regiões e se caracteriza como uma forma de proteção da superfície cutânea contra agressões de microrganismos e fungos (Silva et al., 2019; Lis et al., 2019). Por isso, a variação de $\mathrm{pH}$ da formulação contendo o extrato de $H$. indicum pode ser considerado normal, uma vez que se encontra dentro da faixa de pH correspondentes aos parâmetros ideais para uma formulação cosmética. Relacionando esse parâmetro com a viscosidade da amostra, não foi possível afirmar que a alteração do pH sofreu alguma influência marcante na alteração da viscosidade. 
Tabela 3. Valores de pH obtidos através do teste do estresse térmico da formulação contendo extrato de Heliotropium indicum L., submetido à temperatura ambiente $\left(25^{\circ} \mathrm{C}\right)$ e estufa $\left(45^{\circ} \mathrm{C}\right)$.

\begin{tabular}{c|c|c}
\hline Ciclo de estabilidade $(24 \mathrm{~h})$ & Valor de $\mathrm{pH}\left(25^{\circ} \mathrm{C}\right)$ & Valor de $\mathrm{pH}\left(45^{\circ} \mathrm{C}\right)$ \\
\hline $\mathrm{t}_{0}$ & $6,3 \pm 0,1$ & $6,3 \pm 0,1$ \\
\hline $\mathrm{t}_{1}$ & $6,6 \pm 0,3$ & $6,0 \pm 0,2$ \\
\hline $\mathrm{t}_{2}$ & $5,2 \pm 0,1$ & $5,4 \pm 0,0$ \\
\hline $\mathrm{t}_{3}$ & $5,3 \pm 0,0$ & $5,3 \pm 0,1$ \\
\hline $\mathrm{t}_{4}$ & $5,4 \pm 0,0$ & $5,1 \pm 0,2$ \\
\hline $\mathrm{t}_{5}$ & $5,3 \pm 0,2$ & $5,8 \pm 0,1$ \\
\hline $\mathrm{t}_{6}$ & $5,5 \pm 0,1$ & 5,1 \\
\hline
\end{tabular}

Resultados expressos pela média \pm DP, com nível de significância de $\mathrm{p}<0,05$. Fonte: Autores.

Nesse sentido, os estudos de planejamento e formulação do gel contendo o extrato de $H$. indicum L. foram realizados para avaliar a viabilidade do seu desenvolvimento tecnológico. E a escolha do polímero (hidroxietilcelulose), para incorporação do extrato vegetal, deu-se em razão às suas propriedade físico-químicas como agente de viscosidade, o qual apresenta natureza não-iônica e boa estabilidade quando submetido numa ampla faixa de pH. Logo, resulta em boa compatibilidade com vários insumos farmacêuticos ativos, principalmente em extratos vegetais (Silva Júnior \& Pereira, 2009). O HEC também apresenta facilidade de aplicação e espalhabilidade sobre a pele (Corrêa et al., 2005). Assim, a base gelificante de HEC (1,5\%) permaneceu estável quando submetido ao estresse mecânico e térmico, caracterizando-a em mais um indicativo primário de estabilidade da formulação.

\subsection{Comportamento reológico da formulação}

A avaliação do comportamento reológico do gel contendo o extrato vegetal apresentou um perfil de material fluidificante com características de material pseudoplástico (Figura 1), indicando que a formulação sofreu uma deformação com a tensão de cisalhamento e a sua viscosidade diminuiu. Nesse contexto, o fluxo pseudoplástico é o mais comum nas formulações cosméticas, pois esses materiais têm sua viscosidade aparente diminuída gradualmente à medida que aumenta a tensão de cisalhamento e, portanto sua viscosidade não pode ser expressa por um único valor (Carvalho et al., 2013; Qwist et al., 2019).

Figura 1. Perfil da viscosidade da formulação contendo extrato de Heliotropium indicum L.

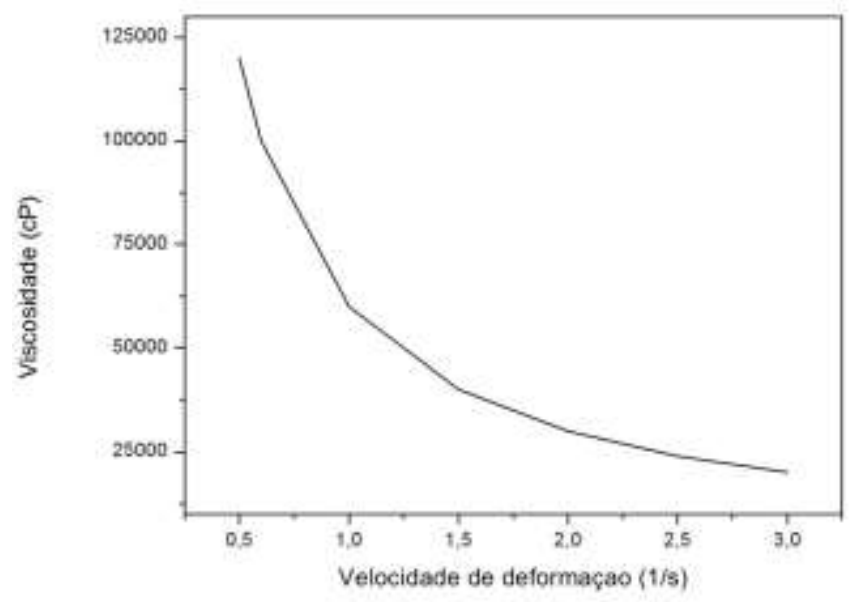

Fonte: Autores. 
Nesse sentido, a pseudoplasticidade é característica de sistemas contendo polímeros em dispersão e sistemas emulsionados semissólidos. Segundo Ferreira et al. (2016), materiais que apresentam esse perfil possuem estruturas reticulares que podem ser constituídas por aglomerados de moléculas que se atraem ou por uma rede de cadeias poliméricas emaranhadas (quando estão em repouso). O gel também apresentou um comportamento tixotrópico, resultado semelhante foi encontrado por Bonfim et al. (2020) que estudaram as propriedades antifúngicas de uma formulação semissólida para o tratamento da vulvovaginite. Nesse estudo, todas as formulações exibiram tixotropia, as quais foram diretamente influenciadas pela temperatura e pela presença do extrato de própolis.

Nessa senda, um produto com características tixotrópica, em geral, apresenta maior vida de prateleira ("shelf-life"), pois durante seu armazenamento tende a apresentar viscosidade constante, dificultando a separação dos constituintes da formulação. Além disso, a obtenção de formulações para uso tópico com caráter tixotrópico é bastante almejada, pois elas se deformam durante a aplicação, ou seja, tornando-se mais fluídas e facilitando o espalhamento e a recuperação da viscosidade inicial, evitando que o produto escorra sobre a pele (Carvalho et al., 2013; Ferreira et al., 2016).

A Figura 2 mostra uma deformação no comportamento reológico do gel, com presença de uma área de histerese com sobreposição das curvas ascendentes e descendentes. Sinalizando uma recuperação da viscosidade inicial, resultado da presença de um componente viscoelástico na formulação (Qwist et al., 2019). Segundo Silva Jr. e Pereira (2009), tal comportamento pode ser atribuído ao hidroxietilcelulose, devido sua propriedade doadora de viscosidade.

Figura 2. Comportamento reológico do gel contendo extrato de Heliotropium indicum L.

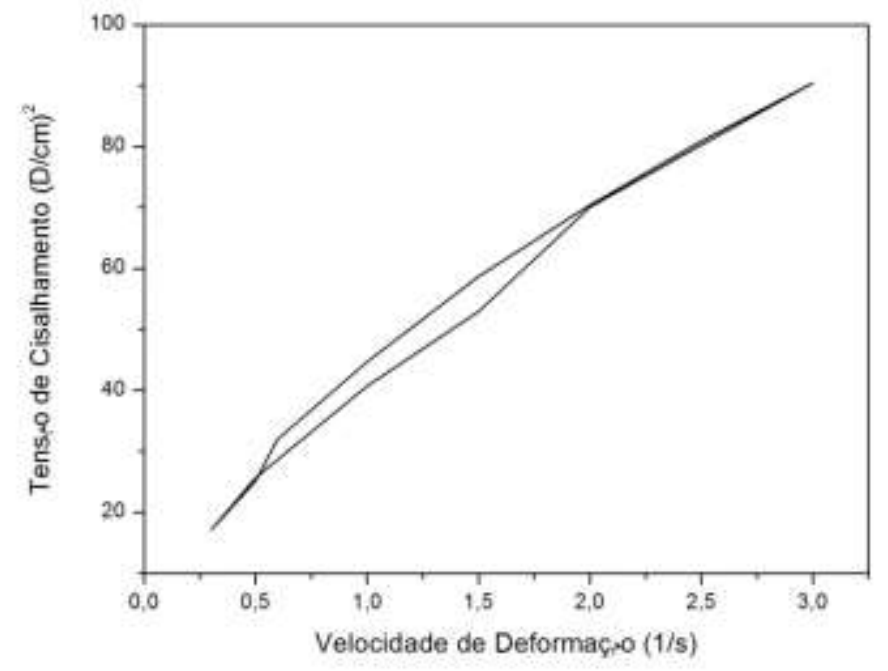

Fonte: Autores.

Também foi possível observar que a curva ascendente da viscosidade está deslocada abaixo da curva descendente (Figura 2), indicando que o material possui uma consistência menor para qualquer taxa de cisalhamento sobre a curva ascendente em relação à curva descendente. Indicando, portanto, que ocorreu uma quebra da estrutura, a qual não se restaurou de imediato após a remoção ou diminuição da tensão de cisalhamento.

\subsection{Avaliação da atividade antimicrobiana}

A atividade antimicrobiana in vitro do gel contendo o extrato de $H$. indicum L. mostrou inibição no crescimento de cepas gram-positivas, porém não foi capaz de inibir as cepas gram-negativas. Portanto, o gel contendo o extrato vegetal de $H$. 
indicum foi capaz de inibir o crescimento da cepa de Staphylococcus aureus nas concentrações de 500 e $250 \mathrm{mg} / \mathrm{mL}$, entretanto, não apresentou atividade frente as cepas de P. aerugiona, E. coli e C. albicans em nenhuma concentração analisada (Tabela 4).

A capacidade de inibição do crescimento bacteriano do extrato de $H$. indicum L. frente à cepa gram-positiva pode estar diretamente relacionada com a estrutura da parede celular desses microrganismos, porque a presença da membrana externa das cepas gram-negativas funciona como uma barreira para certos tipos de antibióticos, enzimas digestivas e metais pesados (Santos et al., 2007; Souza, Pessoa \& Pessoa, 2018).

Tabela 4. Valores médios dos halos de inibição $(\mathrm{mm})$ obtidos pelo método de difusão em disco do gel contendo o extrato de Heliotropium indicum L. em diferentes concentrações.

\begin{tabular}{l|c|c|c|c}
\hline & \multicolumn{3}{|c}{ Concentração (mg/mL) } \\
Cepas de micronganiamos & $\mathbf{5 0 0}$ & $\mathbf{2 5 0}$ & $\mathbf{6 2 , 5}$ \\
\hline Staphylococcus aureus (ATCC 29913) & + & + & - & - \\
\hline Pseudomonas aeruginosas (ATCC & - & - & - & - \\
25853 ) & & & & \\
\hline Escherichia coli (ATCC 25922) & - & - & - & - \\
\hline Candida albicans (ATCC 40175) & - & - & - & - \\
\hline
\end{tabular}

+ inibição; - sem inibição; (média do halo de inibição 土 DPR). Fonte: Autores.

Outros estudos com a espécie Heliotropium ellipticum foi verificado um amplo espectro e uma elevada atividade antimicrobiana, utilizando a fração hexânica do extrato etanólico, constatando que a substância $\beta$-amirina apresentou a maior atividade antimicrobiana, enquanto o acetato de $\beta$-amirina foi o mais ativo para atividade antifúngica (Jain, Singh \& Jain, 2001). Como a espécie em estudo (H. indicum L.) também possui a substância $\beta$-amirina na sua composição (Singh et al., 2005), sugerese que essa substância seja uma das responsáveis pela atividade antimicrobiana apresentada pela formulação contendo o extrato vegetal.

Assim, a importância da formulação contendo o extrato de $H$. indicum L. em apresentar inibição no crescimento da cepa de $S$. aureus está relacionado, principalmente, ao fato dessa espécie ser responsável por causar abscessos e várias infecções piogênicas em humanos e ainda, mais de $90 \%$ dos cocos gram-positivos conterem plasmídeos que codificam a $\beta$-lactamase, ou seja, uma enzima que degrada muitas penicilinas disponíveis para tratamento (Levinson \& Jawetz, 2005). Portanto, os resultados referentes à atividade antimicrobiana do gel contendo o extrato de $H$. indicum frente às cepas de $S$. aureus demonstraram que a espécie vegetal apresentou atividade antimicrobiana, tornando-se relevante no que diz respeito ao desenvolvimento de antimicrobianos para uso tópico.

\section{Conclusão}

Foi possível desenvolver uma formulação tópica contendo o extrato de $H$. indicum L. com manutenção da sua estabilidade físico-química. Nesse sentido, o gel não apresentou desestruturação da cadeia polimérica após submissão à força centrípeta e permaneceu estável após sofrer influência da temperatura $\left(45^{\circ} \mathrm{C}\right)$. Após a incorporação do extrato vegetal na base gelificante, não apresentou alteração significativa no pH, mantendo-se estável e compatível com o pH cutâneo. O gel apresentou um comportamento pseudoplástico e tixotrópico, o que facilita seu espalhamento e evita que o produto escorra sobre a pele. Além, de atividade inibitória, nas concentrações de 500 e $250 \mathrm{mg} / \mathrm{mL}$, frente à cepa de Staphylococcus aureus, vislumbrado, 
assim, o desenvolvimento de um fitoterápico com ação antimicrobiana para uso tópico. Diante disso, é importante dar-se continuidade aos estudos de estabilidade acelerada visando o estabelecimento do prazo de validade do gel fitoterápico, bem como estudo in vitro de permeação cutânea.

\section{Agradecimentos}

Os autores agradecem à Coordenação de Aperfeiçoamento de Pessoal de Nível Superior (CAPES) pelo auxílio na forma de bolsa concedida, e a Fundação de Amparo à Pesquisa do Estado do Pará (FAPESPA) pelo suporte financeiro ao projeto.

\section{Referências}

Alves, M. J., Pereira, M. S. R., Fraga, S. C., Ferreira, I. C., \& Dias, M. I. (2020). Atividade antimicrobiana de extratos naturais e elixires comerciais em patógenos orais: Estudo "in-vitro". Conhecimento Pesquisa Ciências da Saúde, 5.

Ansel, H. C., Popovich, N. G., \& Allen, L. V. J. R. (2013). Formas Farmacêuticas e sistemas de liberação de Fármacos. (9ª ed.): Editora Artmed.

Barros Silva, S., de Oliveira, H. G. B., Gama, G. S. P., Pinheiro, R. E. E., de Souza, J. S. N., \& Chaves, T. P. (2019). Produtos naturais de Momordica charantia L. como moduladores da resistência de Escherichia coli a fármacos antimicrobianos. Agropecuária Ciência do Semiárido, 15 (3), $219-223$.

Bonfim, A. P., Sakita, K. M., Faria, D. R., Arita, G. S., Vendramini, F. A. V. R., Capoci, I. R. G., et al. (2020). Preclinical approaches in vulvovaginal candidiasis treatment with mucoadhesive thermoresponsive systems containing propolis. PLoS One, 15(12), 1-20.

Brasil. (2010). Agência Nacional de Vigilância Sanitária. Farmacopeia Brasileira. (5ed.): Anvisa.

Brasil. (2004). Ministério da saúde. Agência Nacional de Vigilância Sanitária. "Guia para realização de estudos de estabilidade de produtos cosméticos”. Série Temática. Brasília. ANVISA.

Bresslau, S. (2017) Plano de Ação Nacional para Prevenção e Controle da Resistência aos Antimicrobianos. http://www.agricultura.gov.br/../SUZANA.MAPACPRASeminrioSMCBPAsuinocultur amar.

Carvalho, F. C., Calixto, G., Hatakeyama, I. N., Luz, G. M., Gremião, M. P. D., \& Chorilli, M. (2013). Rheological, mechanical, and bioadhesive behavior of hydrogels to optimize skin delivery systems. Drug Development and Industrial Pharmacy, 39(11), $1750-7$.

Corrêa, N. M., Camargo Junior, F. B., Ignácio, R. F., \& Leonardi, G. R. (2005). Avaliação do comportamento reológico de diferentes géis hidrofílicos. Revista Brasileira de Ciências Farmacêuticas, 41(1), 73-78.

Costa, R. S., Camelo, S. R. P., Ribeiro-Costa, R. M., Barbosa, W. L. R., Vasconcelos, F., \& Silva Júnior, J. O. C. (2011). Physical, Chemical and Physicochemical Control of Heliotropium indicum Linn., Boraginaceae, Powder and Tinture. International Journal of Pharmaceutical Science and Research, 2(7), 2211-2216.

Dubey, V., et al. (2007). Dermal and transdermal delivery of an anti-psoriatic agent via ethanolic liposomes. Journal of Control Release, 123(2), 148-154.

Estrela, C. (2018). Metodologia Científica: Ciência, Ensino, Pesquisa. Editora Artes Médicas.

Ferreira, S. B. D. S., Moc, T. D., Borghi-Pangoni, F. B., Junqueira, M. V., \& Bruschi, M. L. (2016). Rheological, mucoadhesive and textural properties of thermoresponsive polymer blends for biomedical applications. Journal of the Mechanical Behavior of Biomedical Materials, 55, 164-178.

Guerrero, M. G. M. (1994). Obtencion y aprovechamento de extractos vegetales de la flora Salvadorena. (2 ${ }^{\mathrm{a}}$ ed.) San Salvador: Editora Universitaria, Universidad de El Salvador.

Isaac, V. L. B., Cefali, L. C., Chiari, B. G., Oliveira, C. C. L. G., Salgado, H. R. N., \& Corrêa, M. A. (2008). Protocolo para ensaios físico-químicos de estabilidade de fitocosméticos. Revista de Ciências Farmacêuticas Básica e Aplicada, 29(1), 81-96.

Jain, S. C., Singh, B., \& Jain, R. (2001). Antimicrobial activity of triterpenoids from Heliotropium ellipticum. Fitoterapia, 72(6), 666-668.

Levinson, W., \& Jawetz, E. (2005). Microbiologia médica e imunologia. (7ª Ed.): Editora Artmed.

Lima, C. G., Vilela, A. F. G., Silva, A. A. S., Piannovski, A. R., Silva, K. K., Carvalho, V. F. M., Musis, C. R. D., Machado, S. R. P., \& Ferrari, M. (2008). Desenvolvimento e avaliação da estabilidade física de emulsões O/A contendo óleo de babaçu (Orbignya oleifera). Revista Brasileira de Farmácia, (3), 239245 .

Lis, C. C., Massuquetto, R. F., da Silva, T. F. B., \& Lubi, N. C. (2019). Development and evaluation of stability of a gel containing caffeine. Revista Saúde e Desenvolvimento, 13(16), 101-119.

Marks, F. O., De Oliveira, T. M. S., Ferreira, G., Dallabrida, M. M., Bisewski, C. G., \& de Souza, P. A. (2020). Infecção do trato urinário: etiologia, perfil de sensibilidade e resistência aos antimicrobianos em hospital pediátrico. Research Society and Development, 9(8), e677985807.

NCCLS (Clinical and Laboratory Standards Institute). Performance Standards for Antimicrobial Disk Susceptibility Tests; Approved Standards. NCCLS document M2-A8 [ISBN 1- 56238-485-6], Wayne, Pennsylvania, 2003a. 
Research, Society and Development, v. 10, n. 5, e32310515068, 2021

(CC BY 4.0) | ISSN 2525-3409 | DOI: http://dx.doi.org/10.33448/rsd-v10i5.15068

OPAS (2017). Organização Pan-americana de Saúde. https://www.paho.org/bra/index.php?option=com_content\&view=article\&id=5664:folha-informativaresistencia-aos-antibioticos\&Itemid=812.

Parekh, J., \& Chanda, S. V. (2007). In vitro antimicrobial activity and phytochemical analysis of some Indian medicinal plants. Turkish Journal of Biology, 31, 53-58.

Qwist, P. K., Sander, C., Okkels, F., Jessen, V., Baldursdottir, S., \& Rantanen, J. (2019). On-line rheological characterization of semi-solid formulations. European Journal of Pharmaceutical Sciences, 12, 36-42.

Rincón-León, H. Á., \& Navarro-Fuentes, K. R. (2016). Tendencias de resistencia antimicrobiana en patógenos aislados de infecciones nosocomiales. Revista Médica del Instituto Mexicano de Seguro Social, 54 (1), 32-41.

Santos, S. C., Ferreira, F. S., Rossi-Alva, J. C., \& Fernandez, L. G. (2007). Atividade antimicrobiana in vitro do extrato de Abarema cochliocarpus (Gomes) Barneby \& Grimes. Revista Brasileira de Farmacognosia, 17(2), 215-219.

Silva, F. V. F., Santos, M. C., Neiva, L. D. B., Oliveira, M. A. C., Leal, B. S., et al. (2019). Development and quality control of an anti-acne gel-cream obtained from the essential oil of Copaífera officinalis L. (copaíba). Revista Eletrônica Acervo Saúde, 30 | e974, 1-10.

Silva Júnior, J. O. C., \& Pereira, N. L. (2009). Avaliação da permeação in vitro de gel fitoterápico contendo extrato seco por nebulização de Shymphytum officinale L. Revista Brasileira de Farmácia, 90(1), 03-09.

Silva, T. C., \& Torres, V. M. (2019). Evelopment and study of accelerated stability of a phytocosmetic containing essential oil of Cordia verbenacea (ervabaleeira). Revista Brasileira de Educação e Saúde, 9 (4), 127-133.

Singh, J. P., Pandey, D. P., Pandey, M. B., Sing, A., \& Singh, R. (2005). Alkaloids of Heliotropium indicum. Journal of the Indian Chemical Society, 82(2), $175-176$.

Souza, T. A. A, Pessoa, F. O. A.. \& Pessoa, M. S. (2018). Produtos naturais com atividade antimicrobiana frente a patógenos de interesse humano. Journal Applied Pharmaceutical Science, 5(3), 8-18.

Stella, A. E., \& de Oliveira, A. F. (2020). Padrões de resistência a antibióticos em enterobactérias isoladas de infecções do trato urinário em gestantes. Research Society and Development, 9(8), e862986337. 